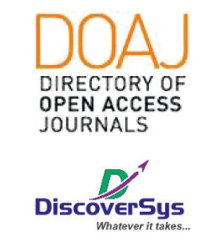

Published by DiscoverSys

\title{
The relationship between neutrophil to lymphocyte ratio (NLR) at admission and GRACE mortality risk score in acute myocardial infarction patient at Tabanan Regency Hospital in 2017
}

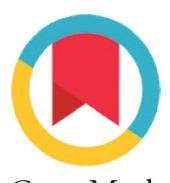

CrossMark

\author{
Gede Wikananda ${ }^{1 *}$, Eka Ariawan², Michelle Husin ${ }^{3}$
}

\begin{abstract}
Background: This study aimed to investigate the role of neutrophil to lymphocyte ratio (NLR) as non-specific and independent laboratory biomarkers when associated with Global Registry of Acute Coronary Events (GRACE) mortality risk score in predicting the morbidity and mortality of patient with acute myocardial infarction (AMI).

Methods: A cross-sectional study design among 103 AMI patients who met inclusion criteria were enrolled in this study. NLR was the ratio of absolute count between neutrophil and lymphocyte. In addition, the GRACE score was a valid instrument in evaluating acute coronary syndrome (ACS). Data were analyzed using SPSS

Results: Correlation test showed a weak positive correlation between NLR value and GRACE risk score $(r=0,388 ; p<0,05)$. Bivariate comparative analysis showed that there was a significant difference in NLR mean in GRACE score group $(F(2,100)=5,215$, $p=0,007)$. Using the least significance different, it was found that there was a significant difference of NLR mean between low and high GRACE score group $\{(\mathrm{p}=0,003 ; 95 \% \mathrm{Cl}=-3,556-(-0,736)\}$. Conclusion: Our study suggests that there was an increase in NLR value proportional to the higher GRACE stratification risk score and there was a significant difference between NLR values in low and high GRACE groups.
\end{abstract} software version 17.

Keywords: NLR, GRACE, Acute Myocardial Infarction

Cite This Article: Wikananda, G., Ariawan, E., Husin, M. 2019. The relationship between neutrophil to lymphocyte ratio (NLR) at admission and GRACE mortality risk score in acute myocardial infarction patient at Tabanan Regency Hospital in 2017. Intisari Sains Medis 10 (1): 205 208. D0I: 10.1556/ism.v10i1.381

${ }^{1}$ General Practitioner at Cardiovascular Department BRSU Tabanan Hospital, Tabanan, Indonesia

${ }^{2}$ Cardiologist at BRSU Tabanan Hospital, Tabanan, Indonesia ${ }^{3}$ General Practitioner at Wangaya Hospital, Denpasar, Indonesia

*Corresponding: Gede Wikananda; General Practitioner at Cardiovascular Department BRSU Tabanan Hospital, Tabanan, Indonesia;

g.wikananda@gmail.com

Diterima : 30-11-2018

Disetujui : 29-03-2019

Diterbitkan : 01-04-2019

\section{INTRODUCTION}

Coronary Heart Disease (CHD) is a condition caused by an inadequate supply of blood and oxygen to the myocardium, resulting in an imbalance between blood needs and supply. This condition can lead to myocardial disturbance ranging from reversible myocardial ischemia to irreversible myocardial death or myocardial infarction marked by increased cardiac biomarkers post attack. ${ }^{1,2}$

As well known, atherosclerosis is an important mechanism in myocardial infarction and the role of inflammation in the process of initiation, progression and plaque de-stabilization in atherosclerosis has been well studied. White blood cell (WBC) and its sub-type are one of the inflammatory markers that have been studied for a long time in cardiovascular disease. Relative neutrophilia and lymphopenia were a potential condition as a predictor of mortality and recurrence in patients with myocardial infarction. The ratio of neutrophils and lymphocyte (NLR) is a combination of two independent markers above which is simple and non-specific to predict the inflammatory state in patients with myocardial infarction as well as predictors of mortality and morbidity. ${ }^{2-5}$

Global Registry of Acute Coronary Events (GRACE) risk score is a validated scoring system used in patients with acute coronary syndrome (ACS) to predict mortality in the hospital. This study studied how the role of NLR as a nonspecific and independent laboratory biomarker in predicting the morbidity and mortality of patient with myocardial infarction when associated with GRACE mortality risk score. ${ }^{6}$

\section{METHODS}

\section{Study Design, Definition and End Points}

This is an observational analytic study with crosssectional study design. The design of this study aims to find the relationship between two variables measured simultaneously at one time. In this study, no follow up required.

This study was conducted in Tabanan regency hospital in March 2018 using patient medical 
records. Samples were taken using total sampling methods, ie patients who came to BRSU Tabanan Hospital Emergency Medical Services (EMS) from January $1^{\text {st }} 2017$, until December $31^{\text {st }} 2017$, diagnosed with Acute Myocardial Infarction (AMI). Samples were obtained using computerized systems that had been integrated with the patients diagnosis using keywords; "acute myocardial infarction" then patient medical records can be obtained. The diagnosis of AMI is obtained based on the criteria of the European Society of Cardiology (ESC) which includes a significant increase in cardiac biomarker with at least one of; symptoms of ischemia, changes in ST segments as well as new or considered new $\mathrm{T}$ waves or new onset LBBB, and progression of pathological Q waves on ECG. The AMI spectrum includes both STEMI and NSTEMI. ${ }^{7}$

Patient's demographic data and data on the variables to be studied were recorded after being obtained through the patient's medical records. Data on variables to be studied include complete blood count value (WBC, neutrophil, lymphocyte) which performed the first time at the admission and data that determined GRACE mortality risk score. NLR is calculated based on neutrophil to lymphocyte count ratio. GRACE scores include; age, heart rate, systolic blood pressure, creatinine, cardiac arrest upon arrival, ST-segment deviation on ECG, cardiac enzyme abnormalities and Killip class. The GRACE score was calculated using the MDCalc application, which was later obtained numerical values and classified as low $(<108)$, intermediate (108-140) and high (>140). ${ }^{3}$

Exclusion criteria include; leukocytosis or current inflammatory condition other than AMI that may interfere with high or low NLR output to GRACE score; patients with acute infections, cancer, hematological disorders, systemic inflammatory conditions (SIRS), immune-mediated disease or autoimmune disorders, chronic kidney disease, and chronic liver disease. ${ }^{3}$ We checked patient medical reports whether they had a history of certain medical condition above and current inflammatory condition.

Table 1. Variable Distribution Based on GRACE score Classification $(\mathrm{N}=103)$

\begin{tabular}{lccc}
\hline \multirow{2}{*}{ Variable } & \multicolumn{3}{c}{ GRACE score } \\
\cline { 2 - 4 } & Low & Intermediate & High \\
\hline Age (Years) & $52,25 \pm 7,71$ & $62,42 \pm 9,16$ & $72,55 \pm 12,35$ \\
Males (N(\%)) & $48(84,2)$ & $20(83,3)$ & $19(86,4)$ \\
NLR & $3,18 \pm 2,73$ & $4,53 \pm 2,58$ & $5,33 \pm 3,32$ \\
\hline
\end{tabular}

NLR: Neutrophil to lymphocyte ratio; P-value: statistically significant if less than 0.05

\section{Data Analysis}

Statistical analysis was performed using SPSS softwareversion 17.0 (SPSS Inc., Illinois). Categorical variables are presented in the table, in percentage form while numerical variables are presented in the table in the mean \pm standard deviation. Variables distribution based independent variable category is also presented. Normality and homogeneity tests were performed on independent variables using the Kolmogorov-Smirnov test and Levene test. To assess the magnitude of the correlation between 2 variables, the Pearson correlation test was performed. Comparative test to measure the mean of NLR value in GRACE score groups is done using one-way ANOVA if eligible. If the results are significant, the post-hoc test performed. Chi-square test also performed to determine whether there was a significant difference in the proportion of GRACE scores between women and men, considering sexrelated disparities exist in presentation, treatment and outcome patient with AMI and not a component of GRACE score. ${ }^{8}$

\section{RESULTS}

From 117 samples with AMI, 103 samples met the inclusion criteria. From the total sample, more than half $(84.5 \%)$ were men with a mean sample age $58.95 \pm 12.28$ years. The average ratio of neutrophil and lymphocyte was $3.96 \pm 2.95$. Based on GRACE risk score, 57 (55.3\%) samples with a low GRACE risk score, $24(23.3 \%)$ samples with an intermediate GRACE risk score, and 22 (21.4\%) samples with a high GRACE risk score. Variables distribution based on GRACE risk score classification can be seen in the Table 1.

From the normality test results using Kolmogorov-Smirnov, $\mathrm{p}$-value obtained $=0.239(\mathrm{p}>$ 0.05 ), which mean the average data of NLR based on GRACE score has a normal distribution. The result of homogeneity test using Levene test showed p-value $=0,126(p>0,05)$ this means the value of NLR based on GRACE score has homogeneous variation, a bivariate test can be continued using parametric test using Pearson correlation test which can be seen on the following table (Table 2).

From the Pearson correlation test, there was a weak positive correlation between NLR value and GRACE risk score $(r=0,388 ; \mathrm{p}<0,05)$. This positive correlation means an increasing value of NLR will be followed by an increase in GRACE score. In this study, one-way ANOVA test was performed to compare the mean of NLR value in GRACE score group classification (low, intermediate, and high). This test showed that there was a significant difference in NLR mean in GRACE score group ( $F$ $(2,100)=5,215, \mathrm{p}=0,007)$ (Table 3$)$. 
Table 2. Bivariate Test Results between NLR and GRACE score using Pearson Correlation Test $(\mathrm{N}=103)$

\begin{tabular}{lccccc}
\hline \multirow{2}{*}{ Variable } & \multicolumn{3}{c}{ GRACE score } & \multirow{2}{*}{ R } & p-value \\
\cline { 2 - 4 } & Low & Intermediate & High & & \\
\hline NLR & $3,18 \pm 2,73$ & $4,53 \pm 2,58$ & $5,33 \pm 3,32$ & 0,388 & 0.000 \\
\hline
\end{tabular}

NLR: Neutrophil to lymphocyte ratio; P-value: statistically significant if less than 0.05

Table 3. Bivariate Test Results between NLR and GRACE Score Using One Way ANOVA Test ( $\mathrm{N}=103)$

\begin{tabular}{lccc}
\hline \multicolumn{1}{c}{ NLR } & Df & F & p-value \\
\hline Between Groups & 2 & 5.215 & 0.007 \\
Within Groups & 100 & & \\
Total & 102 & & \\
\hline
\end{tabular}

NLR: Neutrophil to lymphocyte ratio; P-value: statistically significant if less than 0.05 ; Df: Degree of freedom

Table 4. Bivariate Test Results using Chi-Square between sex and GRACE Score $(\mathrm{N}=103)$

\begin{tabular}{lccccc}
\hline \multirow{2}{*}{ Sex } & \multicolumn{3}{c}{ GRACE } & \multirow{2}{*}{ R } & \multirow{2}{*}{$\mathbf{p}$} \\
\cline { 2 - 4 } & Low & Intermediate & High & & \\
\hline Males (N (\%)) & $48(84,2)$ & $20(83,3)$ & $19(86,4)$ & \multirow{2}{*}{0,07} & 0,958 \\
Females (N (\%)) & $9(15,8)$ & $4(16,7)$ & $3(13,6)$ & & \\
\hline
\end{tabular}

This significant result is a requirement to perform post hoc test. Using the least significance different (LSD), it was found that there was a significant difference of NLR mean between low and high GRACE score group. $\{(\mathrm{p}=0,003 ; 95 \% \mathrm{CI}$ $=-3,556-(-0,736)\}$.

From bivariate test using chi-square, it showed that there was no significant difference in GRACE score proportion between a group of women and men ( $p>0,05)$. In other words, there is no relationship between sex and GRACE score.

\section{DISCUSSION}

White blood cells play an important role in the underlying inflammatory process of atherosclerosis. Neutrophils have a role in acute plaque disruption and blockage due to the thrombus. Neutrophils were secreted by bone marrow in response to acute inflammatory process. The interaction between neutrophils and endothelial tissues has been hypothesized to cause increased damage to endothelium and made plaque more vulnerable by the release of proteolytic enzymes, arachidonic acid, and superoxide radicals. ${ }^{9,10}$ Meanwhile lymphocytes play a role in early plaque formation as a response to physiological stress. It depletes secondary to stressrelated cortisol hormone release in AMI. During the inflammatory process, there is an increasing lymphocyte undergo apoptosis. ${ }^{10,11}$ Neutrophilia and lymphopenia both are a condition associated with the severity or increased mortality related to AMI. ${ }^{9,12}$

NLR, a ratio of 2 independent markers above has the best prognostication value in AMI patients compared with other routine hematologic parameters in predicting future cardiovascular events and their severity., ${ }^{4,10,12}$ GRACE score, a validated scoring system, is used to assess mortality risk in AMI patients. The magnitude of GRACE score is proportional to increase the risk of mortality in AMI. ${ }^{6}$ NLR literature suggest this biomarker may be used as an additional independent predictor in addition to the GRACE score in predicting output in AMI patients.,

This study shows that there is a relation between NLR value and GRACE score. Bivariate analysis using Pearson correlation test shows there is a weak positive correlation between NLR value and GRACE score classification. This result is consistent with the theory that WBC plays a vital role in acute atherosclerosis in myocardial infarct manifestations. The higher WBC are also associated with poor outcomes in AMI. ${ }^{4.8}$ Poor outcomes in AMI can be predicted using GRACE scores so that higher WBC are presented in NLR value, proportional with higher GRACE score stratification or value. ${ }^{11,12}$

From comparison test using one-way ANOVA, there is a significant mean difference of NLR in GRACE group. Post hoc test shows us the significant results are in the low and high GRACE group. From those results we can conclude in this study the significant results according to NLR value in GRACE score group can be divided into 2, low and high.

Gender, one of the independent demographic variables toward GRACE score, is a variable that has a role from the initial presentation to the prognosis of the patient with AMI. In this research, we found that there is no relationship between sexes with GRACE score. This is similar to I Gong et al., research which states that the lack of interaction between GRACE score and sexes suggest that sexbased modification is not required. ${ }^{8}$

There are several limitations to this study and should be assessed in future studies. This include there is does not rule out the possibility of falsepositive results or increasing NLR value in any condition non-related to AMI. Regardless of the exclusion criteria made based on patient medical history and brief finding of an acute inflammatory state other than AMI on the medical report, other more specific supporting examination is needed to rule out those circumstances in the future study. 
Other supporting studies with larger samples also need in future to re-assess the power of correlation result and the stratification of GRACE scores based on NLR value due to significant results on comparison test only exist in low and high groups.

\section{CONCLUSION}

In this study, there was an increase in NLR value proportional to the higher GRACE stratification risk score, and there was a significant difference between NLR values in GRACE groups, namely between low and high groups. Gender, an important variable strongly associated with ACS prognosis, seems not having a significant role in GRACE score values. NLR would be an independent, simple, inexpensive and accurate new early predictor of the increasing value of GRACE scores in AMI patient.

\section{ETHICAL CLEARANCE}

Ethical approval has been obtained from the ethics of committee prior to the study conducted

\section{CONFLICT OF INTEREST}

The authors declare that there is no conflict of interest regarding manuscript

\section{FUNDING}

The authors are responsible for the study funding without the involvement of grants, scholarship, or any other resources of funding

\section{AUTHOR CONTRIBUTION}

All authors are equally contributed in this study from main concept, statistical analysis, until data synthesis and research report.

\section{REFERENCE}

1. Ramrakha, P, Hill, J,. Oxford Handbook of Cardiology: Coronary Artery Disease. $1^{\text {st }}$ ed. USA: Oxford University Press. 2006.

2. Antman EM, Braunwald E. 2012. ST-elevation myocardial infarction: pathology, pathophysiology, and clinical features. Dalam: Libby P, Bonow RO, Mann DL, Zipes DP, penyunting Braunwald's heart disease: a textbook of cardiovascular. Edisi ke-9. Philadelphia: Elsevier Sunders

3. Can Oncel R, Ucar M, Karakas MS, et al; Relation of neutrophil-to-lymphocyte ratio with GRACE risk score to in-hospital cardiac events in patients with ST-Segment elevated myocardial infarction. Clinical and applied thrombosis/hemostasis. 2015;21(4):383-388.

4. Yalcinkaya E, Yuksel UC, Celik M, et al; Relationship between neutrophil-to-lymphocyte ratio and electrocardiographic ischemia grade in STEMI. Arq Bras Cardiol. 2014

5. Gul U, Hussain S, Munir R, et al: Neutrophil lymphocyte ratio: a prognostic marker in acute ST Elevation myocardial infarction. Journal of the college of physicians and surgeons.2017;27(1):4-7

6. Perhimpunan dokter spesialis kardiovaskular indonesia. 2015. Pedoman Tatalaksana Sindrom Koroner Akut. Jurnal Kardiologi Indonesia.

7. European Society of Cardiology. Third universal definition of myocardial infarction. 2012; 33:2551-2567

8. I Gong, Gosman SG, Brieger D et al: GRACE risk score: sex based validity of in hospital mortality prediction in Canadian patient with acute coronary syndrome. Canadian journal of cardiology.2016;32(2016):S67-S307

9. Balta S, Celik T, Mikhailidis D T et al: The relation between atherosclerosis and neutrophil-lymphocyte ratio. Clinical and applied thrombosis/hemostasis.2016;22(5):405-411

10. Oner I, Koklu E, Arslan S et al: Association of neutrophil/ lymphocytes ratio with plaque morphology in patients with asymptomatic intermediate carotid artery stenosis.2016;46(5)699-705

11. He J, Li J, Wang Y, et al: Neutrophil-to-lymphocyte ratio (NLR) predicts mortality and adverse-outcomes after ST-segment elevation myocardial infarction in Chinese people. Int J Clin Exp Pathol.2014;7(7):4045-4056

12. Korkmaz A, Yildiz A, Gunes H, et al: Utility of neutrophillymphocyte ratio in pedicting troponin elevation in the emergency department setting. Clinical and applied thrombosis/hemostasis. 2015; 21(7):667-671

13. Prabawa IPY, Bharghah A, Liwang F, Tandio DA, Tandio AL, Lestari AAW, Budiana ING, Manuaba IBAP. Pretreatment Neutrophil-to-Lymphocyte ratio (NLR) and Platelet-to-Lymphocyte Ratio (PLR) as a Predictive Value of Hematological Markers in Cervical Cancer. Asian Pacific Journal of Cancer Prevention, 2019; 20(3).

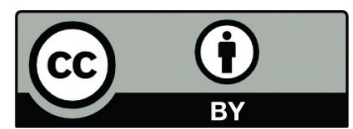

This work is licensed under a Creative Commons Attribution 